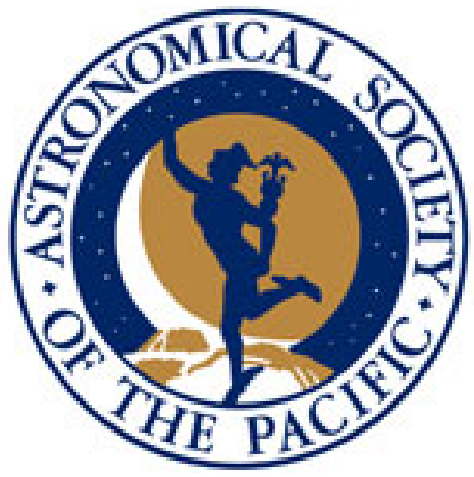

Precipitable Water Vapor above Dome A, Antarctica, Determined from Diffuse Optical Sky Spectra

Author(s): Geoff Sims, Michael C. B. Ashley, Xiangqun Cui, Jon R. Everett, LongLong Feng, Xuefei Gong, Shane Hengst, Zhongwen Hu, Craig Kulesa, Jon S. Lawrence, Daniel M. LuongVan, Philippe Ricaud, Zhaohui Shang, John W. V. Storey, Lifan Wang, Huigen Yang, Ji Yang, Xu Zhou, Zhenxi Zhu

Reviewed work(s):

Source: Publications of the Astronomical Society of the Pacific, Vol. 124, No. 911 (January 2012), pp. 74-83

Published by: The University of Chicago Press on behalf of the Astronomical Society of the Pacific

Stable URL: http://www.jstor.org/stable/10.1086/664077

Accessed: 15/04/2012 20:07

Your use of the JSTOR archive indicates your acceptance of the Terms \& Conditions of Use, available at http://www.jstor.org/page/info/about/policies/terms.jsp

JSTOR is a not-for-profit service that helps scholars, researchers, and students discover, use, and build upon a wide range of content in a trusted digital archive. We use information technology and tools to increase productivity and facilitate new forms of scholarship. For more information about JSTOR, please contact support@jstor.org. 


\title{
Precipitable Water Vapor above Dome A, Antarctica, Determined from Diffuse Optical Sky Spectra
}

\author{
Geoff Sims, ${ }^{1}$ Michael C. B. Ashley, ${ }^{1}$ Xiangqun Cui,${ }^{2}$ Jon R. Everett, ${ }^{1}$ Longlong Feng,,${ }^{3,4}$ Xuefei Gong,,${ }^{2,4}$ \\ Shane Hengst, ${ }^{1}$ Zhongwen Hu, ${ }^{2,4}$ Craig Kulesa, ${ }^{5}$ Jon S. Lawrence, ${ }^{6,7}$ Daniel M. Luong-Van, ${ }^{1}$ \\ Philippe Ricaud, ${ }^{8}$ Zhaohui Shang, ${ }^{4,9}$ John W. V. Storey, ${ }^{1}$ Lifan Wang, ${ }^{3,4,10}$ \\ Huigen Yang, ${ }^{4,11}$ Ji YANG, ${ }^{3}$ XU Zhou, ${ }^{4,12}$ AND ZhenXI ZHU ${ }^{3,4}$ \\ Received 2011 November 6; accepted 2011 December 7; published 2012 January 30
}

\begin{abstract}
The high altitudes and extremely low temperatures of the Antarctic plateau result in an exceedingly low atmospheric water vapor content. In this article we estimate the precipitable water vapor at Dome A using optical spectra of the diffuse solar irradiance in the zenith direction. The spectra were obtained from the Nigel spectrometer at Dome A during 2009. We find that the Nigel spectra contain sufficient information to extract daily average water vapor estimates, which agree with satellite observations to within $\pm 0.22 \mathrm{~mm}$. Finally, we use these water vapor estimates to model the optical and near-IR transmission (between $700 \mathrm{~nm}$ and $2.5 \mu \mathrm{m}$ ) of Dome A to demonstrate significant advantages gained in this wavelength range and compare it with that of Paranal and Chajnantor, two midlatitude observatories.
\end{abstract}

Online material: color figures

\section{INTRODUCTION}

Water vapor is one of the main absorbers of infrared radiation in the Earth's atmosphere. The amount of water vapor present in a column of atmosphere, known as precipitable water vapor (PWV), ultimately determines the suitability of an astronomical site for infrared (IR) and near-IR observations. The benefits of locations on the Antarctic plateau with respect to IR astronomy have been recognized for some time (e.g., Lawrence 2004), with Dome A $\left(\mathrm{S} 80.37^{\circ}, \mathrm{E} 77.53^{\circ}\right)$, the highest point on the plateau, predicted to have the lowest PWV of the few Antarctic plateau sites that are currently developed and

\footnotetext{
${ }^{1}$ School of Physics, University of New South Wales, Sydney NSW 2052, Australia; g.sims@unsw.edu.au.

${ }^{2}$ Nanjing Institude of Astronomical Optics \& Technology, Nanjing 210042, China.

${ }^{3}$ Purple Mountain Observatory, Nanjing 210008, China.

${ }^{4}$ Chinese Center for Antarctic Astronomy, China.

${ }^{5}$ Department of Astronomy and Steward Observatory, University of Arizona.

${ }^{6}$ Department of Physics and Astronomy, Macquarie University, Sydney NSW 2109, Australia.

${ }^{7}$ Australian Astronomical Observatory, Sydney NSW 1710, Australia.

${ }^{8}$ Universite Paul Sabatier, Laboratoire d'Aéologie, Observatoire de MidiPyrénées, Toulouse, France.

${ }^{9}$ Tianjin Normal University, Tianjin 300074, China.

${ }^{10}$ Department of Physics and Astronomy, Texas A\&M University, College Station 77843

${ }^{11}$ Polar Research Institute of China, Shanghai 200136, China

12 National Astronomical Observatories, Chinese Academy of Science, Beijing 100012, China.
}

operationa $1^{13}$ (Saunders et al. 2009). Such extremely low PWV levels have already been recorded from ground-based observations using Pre-HEAT instrument (so-called because it is a precursor to the High Elevation Antarctic Terahertz; Kulesa et al. 2008b) during 2008 (Kulesa et al. 2008a; Yang et al. 2010).

Various methods have been successfully employed to determine the atmospheric PWV content from the ground. These range from simple (even handheld) solar pointing photometers (e.g., Halthore et al. 1997; Raj et al. 2004) to dedicated microwave radiometers that are sensitive to the $183 \mathrm{GHz}$ water vapor line (e.g., Wiedner et al. 2002; Emrich et al. 2009; Ricaud et al. 2010). Another option is to use radiative transfer codes to fit atmospheric models to absorption lines in high-resolution IR stellar spectra (e.g., Querel et al. 2008, 2011); this has also been applied to low-resolution stellar spectra in the optical regime (e.g., Dempsey et al. 2004; Patat et al. 2011). Finally, GPS meteorology techniques have been widely used whereby the PWV can be calculated by analyzing the delay in the microwave signal from a GPS satellite to a ground receiver. This delay is a function of temperature, pressure, and water vapor content (Bevis et al. 1992, 1994). GPS techniques have the advantage of high temporal resolution and the ability to be analyzed in retrospect, and they are able to be utilized in remote locations (e.g., Suparta et al. 2008; Thomas et al. 2011). With accuracies

\footnotetext{
${ }^{13}$ Ridge A, a mere $144 \mathrm{~km}$ from Dome A, is predicted to have the lowest PWV of anywhere on the plateau, with the first expedition expected to visit the site in 2012 January (Storey et al. 2011).
} 
of 1-2 mm, GPS meteorology can be used for temperate sites and even coastal Antarctic stations, but is not suitable for use over the Antarctic plateau, where typical PWV amounts are consistently submillimeter.

In this article we review a number of satellites available for Antarctic PWV retrieval, and we present measurements made with the Nigel $^{14}$ spectrometer of the water vapor absorption in the atmosphere above Dome A. The measurements are made from spectra of diffuse scattered sunlight integrated over a large area of sky. Because the illuminating source (i.e., the Sun) is not entirely behind the absorbing material (as would be the case for a direct absorption measurement), it is effectively a distributed source embedded in the absorbing medium. As such, a more complicated retrieval method is necessary. To our knowledge, Kiedron et al. (2003) provide the only previous documented attempt to do this and had remarkable success. Typical water vapor columns at their site (in Alaska), however, are at least an order of magnitude greater than those found on the Antarctic plateau.

\section{SATELLITE PWV RETRIEVAL}

A number of satellite instruments exist whose measurements allow the derivation of PWV over the Antarctic plateau. Three such instruments are reviewed and compared in this section.

\subsection{MHS/NOAA-18}

The Microwave Humidity Sounder (MHS) is a five-channel microwave instrument on the NOAA-18 polar-orbiting satellite launched in 2005. Developed by NASA for the National Oceanic and Atmospheric Administration (NOAA), the satellite hosts an array of instruments to form a complete meteorological space platform. It has a Sun-synchronous orbit at $98.7^{\circ}$ inclination with a period of 102 minutes at $854 \mathrm{~km}$ altitude. ${ }^{15}$

The satellite passes over Dome A 5 times per day, with a circular field of view at nadir of approximately $16 \mathrm{~km}$ in diameter. MHS has four humidity channels in the $157 \mathrm{GHz}$ to $190 \mathrm{GHz}$ range and a surface-viewing window channel at $89 \mathrm{GHz}$ (Bonsignori 2007).

Level-2 data are provided by the Comprehensive Large Array-data Stewardship System (CLASS),${ }^{16}$ an electronic library of NOAA environmental data, which include calibrated brightness temperature images in the various bands near or in the $183 \mathrm{GHz}$ water vapor line. By comparing the brightness temperatures of each of the three main MHS bands, a PWV calculation can be made (Miao et al. 2001):

\footnotetext{
${ }^{14}$ Nigel is the name given to the spectrometer (Kenyon et al. 2006).

15 See http://www.oso.noaa.gov/poesstatus/spacecraftStatusSummary.asp? spacecraft $=18$.

${ }^{16}$ See http://www.class.noaa.gov.
}
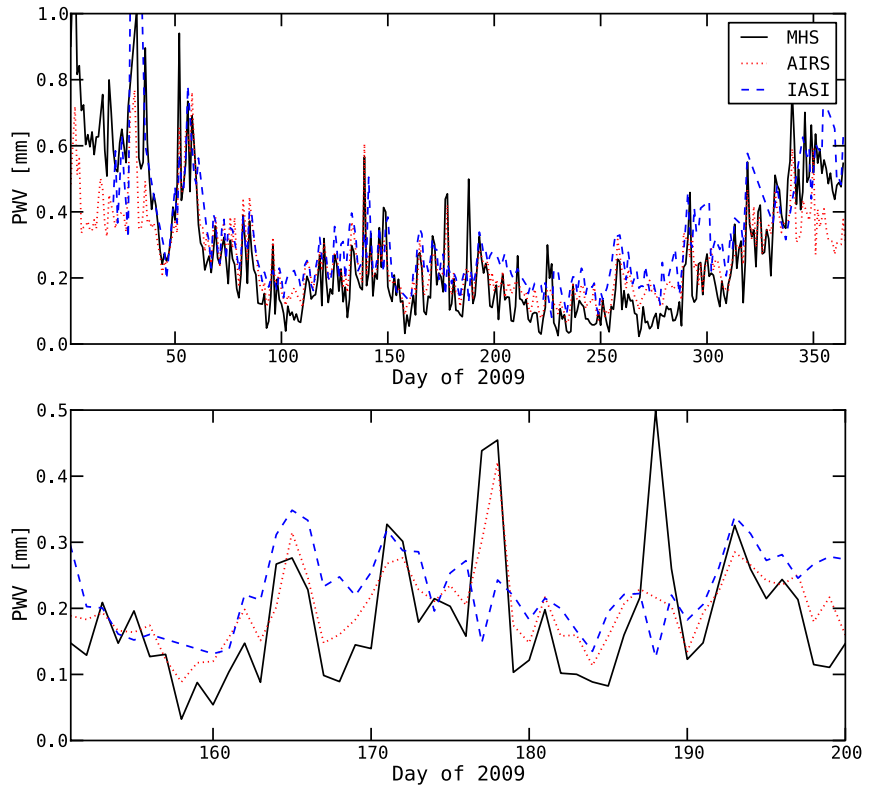

FIG. 1.-Comparison of satellite PWV retrievals for Dome A in 2009. Values plotted are daily averages. Top: Spans the entire year. Bottom: Zooms in on a 50 day period during winter. See the electronic edition of the $P A S P$ for a color version of this figure.

$\operatorname{PWV}(\mathrm{mm})=\cos \theta \times\left(C_{0}+C_{1} \log \left(\frac{T_{5}-T_{4}-b_{45}}{T_{4}-T_{3}-b_{34}}\right)\right)$,

where $\theta$ is the satellite zenith angle, $T_{N}$ is the brightness temperature of channel $N, b_{i j}$ are biases related to the temperature and humidity profiles of the atmosphere, and $C_{0}$ and $C_{1}$ are constants dependent on surface emissivities. The biases and constants were determined by zero-point calibration with observations using the Pre-HEAT instrument (Kulesa et al. 2008b) during 2008 (Yang et al. 2010).

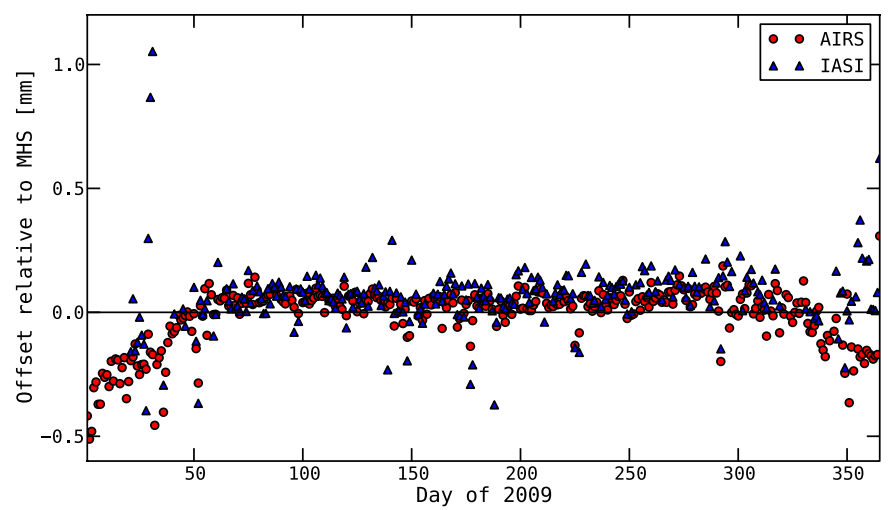

FIG. 2.-Systematic deviations of satellite PWV retrievals for Dome A in 2009. See the electronic edition of the PASP for a color version of this figure. 


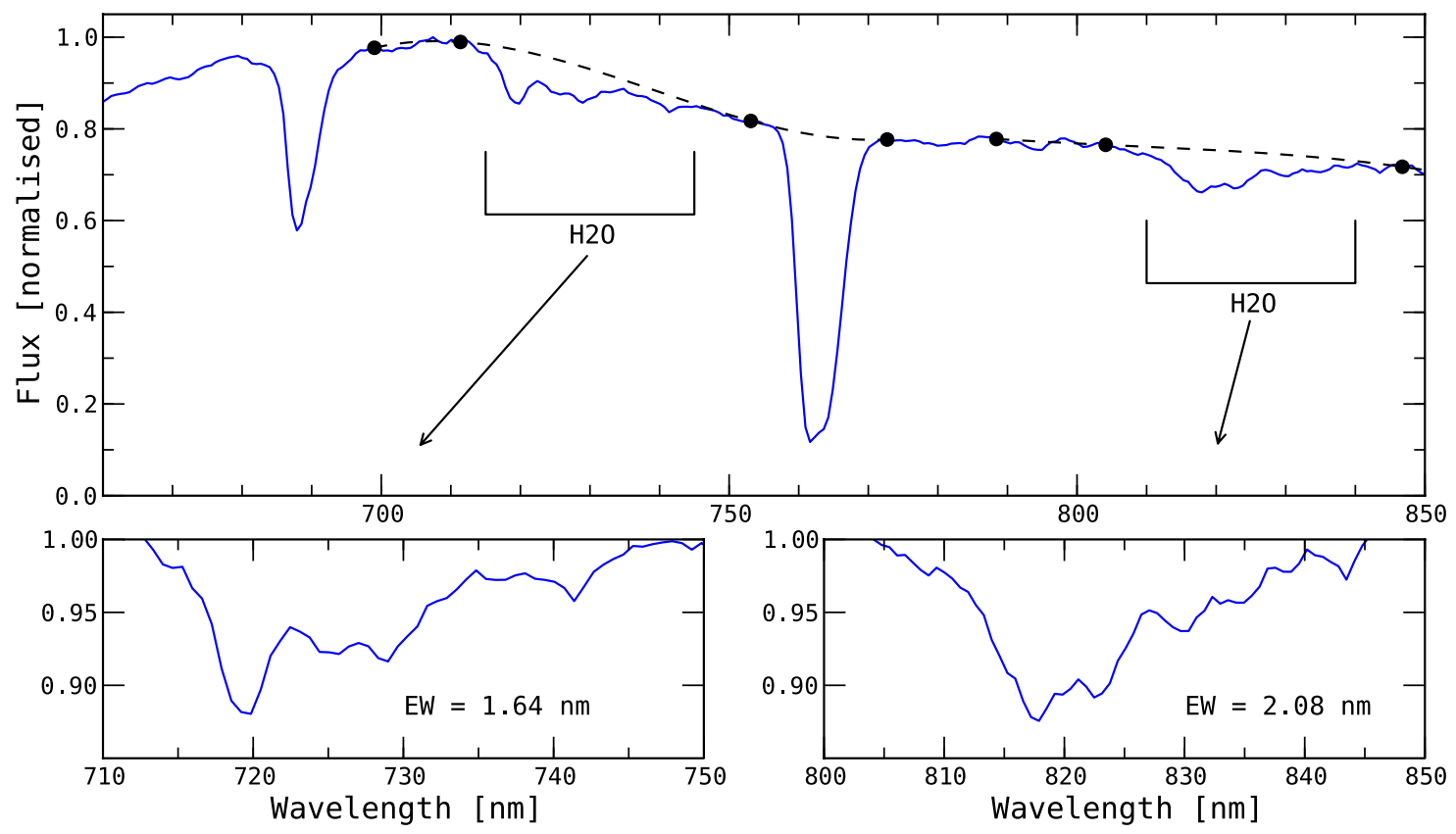

FIG. 3.-Equivalent width calculation (bottom) from a sample Nigel spectrum (top; solid line). Also shown in the upper plot is the continuum approximation (dashed line) and interpolation points (circles). Interpolation points were chosen outside of the oxygen and water absorption bands, where no absorption is expected. The observation was taken on day 68 (during summer), when the PWV was relatively high $(\sim 0.27 \mathrm{~mm})$. See the electronic edition of the PASP for a color version of this figure.

\subsection{IASI/MetOp-A}

The Infrared Atmospheric Sounding Interferometer (IASI) is an instrument of the space platform MetOp-A launched in 2006. The platform has a Sun-synchronous polar orbit inclined $98.7^{\circ}$ and crosses the equator (descending node) at 09:30 local solar time at an altitude of about $815 \mathrm{~km}$. The time for an orbit is 101 minutes with a cycle of 29 days.

IASI is a high-resolution sounder working in the infrared domain that measures temperature and humidity with accuracies better than $1 \mathrm{~K}$ and $10 \%$, respectively, for numerical weather prediction and climate models. The IASI field of view consists of four circular pixels of $0.8^{\circ}$ of angular diameter, corresponding to $12 \mathrm{~km}$ at the surface at nadir. A cross-track angle of sight of $\pm 48.3^{\circ}$ allows a global coverage of the Earth twice a day. Based on a Michelson interferometer, IASI is an accurately calibrated Fourier transform spectrometer covering the 3.6-15.5 $\mu \mathrm{m}$ spectral range with 8461 spectral channels and a resolution between 0.35 and $0.5 \mathrm{~cm}^{-1}$ (Hébert et al. 2004; Schlüssel et al. 2005).

Geophysical level-2 preoperational data are provided by EUMETSAT. ${ }^{17}$ They produce near-real-time vertical profiles of temperature and humidity on a vertical pressure grid (Schlüssel et al. 2005). This grid is converted into an altitude grid by considering the hydrostatic equilibrium and the surface pressure and temperature.

${ }^{17}$ See http://oiswww.eumetsat.org/WEBOPS/eps-pg/IASI-L2/IASIL2-PG0TOC.htm.
A $2^{\circ} \times 2^{\circ}$ bin centered on Dome $\mathrm{A}$ is considered for the study. Only IASI data without cloud contamination in the line of sight are used. This restriction explains why data may not be available for some particular days.

\subsection{AIRS/Aqua}

The Atmospheric Infrared Sounder (AIRS) is an instrument of the space platform NASA EOS Aqua launched in 2002. Together with the Advanced Microwave Sounding Unit and the Humidity Sounder for Brazil, they form an integrated crosstrack scanning temperature and humidity sounding system. The platform has a Sun-synchronous polar orbit with 14 orbits per day and equatorial crossing at 13:30 local solar time ascending node, at the altitude of about $705 \mathrm{~km}$. A cross-track angle of sight of $\pm 49^{\circ}$ allows eight overpasses of Dome A each day with a resolution of $13.5 \mathrm{~km}$ at nadir and $41 \times 21.4 \mathrm{~km}$ at the scan extremes. $^{18}$

AIRS is a high spectral resolution spectrometer working in the thermal infrared that measures humidity with an accuracy better than $20 \%$ in layers $2 \mathrm{~km}$ thick in the troposphere. The primary spectral calibration of the AIRS spectrometer, covering the $3.7-15.4 \mu \mathrm{m}$ spectral range with 2378 spectral channels, is based on the cross-correlation between spectral features observed in the upwelling radiance spectrum with precalculated spectra (Aumann et al. 2003).

\footnotetext{
${ }^{18}$ See http://airs.jpl.nasa.gov/instrument/specs/.
} 
TABLE 1

InPut Parameters to Bird \& Riordan (1986) Diffuse Irradiance Model

\begin{tabular}{lclc}
\hline \hline \multicolumn{1}{c}{ Parameter } & Value & \multicolumn{1}{c}{ Source } & Location \\
\hline Temperature $\ldots \ldots \ldots \ldots \ldots \ldots$ & $-50^{\circ} \mathrm{C}^{\mathrm{a}}$ & Australian Antarctic Division $^{\mathrm{b}}$ & Dome A \\
Surface pressure $\ldots \ldots \ldots \ldots \ldots$ & $570 \mathrm{mbar}$ & Australian Antarctic Division & Dome A \\
Ozone column $\ldots \ldots \ldots \ldots \ldots$. & $0.15 \mathrm{~cm}$ & Commonwealth Scientific and Industrial Research Organization ${ }^{\mathrm{c}}$ & $\sim$ Dome A \\
Aerosol optical depth, $\tau_{a} \ldots \ldots$ & 0.02 & Stone (2002) & South Pole \\
Turbidity exponent, $\alpha \ldots \ldots \ldots$ & 1.65 & Six et al. (2005) & Dome C \\
Surface albedo, $r_{g} \ldots \ldots \ldots \ldots$ & 0.85 & Laine (2008) & $\sim$ Dome A \\
\hline
\end{tabular}

${ }^{a}$ The values are chosen based on typical values observed on the Antarctic plateau.

b See http://www.antarctica.gov.au/science/ice-ocean-atmosphere-and-climate/glaciology-research/antarctic-weather/dome-adetails.

${ }^{\mathrm{c}}$ See http://www.environment.gov.au/atmosphere/ozone/ozone-hole/image2009.html.

The level-3 geophysical parameters (version 5) averaged daily and binned into $1^{\circ} \times 1^{\circ}$ grid cells are provided by NASA on Giovanni, ${ }^{19}$ an online application developed by the Goddard Earth Sciences Data and Information Services Center (Susskind et al. 2009). As for IASI, the profiles of temperature and humidity on a vertical pressure grid are converted into an altitude grid by considering the hydrostatic equilibrium and the surface pressure and temperature.

\subsection{Comparison}

A comparison of the three instruments is shown in Figure 1. Although the satellites generally agree, there are a number of obvious deviations, particularly over the summer months when atmospheric PWV is higher. The systematic deviations are highlighted in Figure 2, where the MHS values were chosen as a base for comparison.

Both AIRS and IASI show significant deviations for days 0-50 and 300-365 (AIRS lower; IASI higher). During days 50-300, AIRS and IASI show smaller systematic overestimations of PWV (compared with that of MHS) by medians of $0.046 \mathrm{~mm}$ and $0.075 \mathrm{~mm}$, respectively.

Previous ground-based PWV observations at Dome A have shown an exceptionally good correlation with the MHS satellite data (Yang et al. 2010). At Dome C, comparisons with radiosondes and a ground-based microwave radiometer show IASI and AIRS systematic dry biases of around 5\% (Ricaud et al. 2011) and 10\% (Carminati et al. 2012, in preparation), respectively.

For consistency with prior results used to determine the PWV at Dome A (i.e., Saunders et al. 2009; Yang et al. 2010), the MHS/NOAA-18 instrument is chosen as a benchmark for comparison against the ground measurements in $\S 4$.

\section{INSTRUMENTATION}

The ground-based instrument used for this study is the Nigel spectrometer (Sims et al. 2010), one of the various site-testing

\footnotetext{
${ }^{19}$ See http://disc.sci.gsfc.nasa.gov/giovanni/overview/index.html.
}

and scientific instruments on PLATO (Plateau Observatory), a self-powered robotic observatory that runs autonomously at Dome A (Lawrence et al. 2009a). Nigel continuously records sky spectra over a wide-field $\left(\sim 25^{\circ}\right.$ diameter or $\left.491 \mathrm{deg}^{2}\right)$ from approximately 300 to $850 \mathrm{~nm}$ in three fixed directions on the sky (north at $40^{\circ}$ altitude, west at $71.5^{\circ}$ altitude, and the zenith). The achieved spectral resolution is $3 \mathrm{~nm}$ FWHM at $500 \mathrm{~nm}$. Although the primary aim of Nigel is to measure night sky brightness, the wavelength range includes two water vapor absorption features that, along with the continuous nature of Nigel's observations (including times when the Sun is above the horizon), make it a suitable instrument to probe the PWV above Dome A.

The two $\mathrm{H}_{2} \mathrm{O}$ bands of interest, designated $\alpha$ and $\beta$ (Rozenberg 1966), are found at approximately $710-740 \mathrm{~nm}$ and 800-840 nm, respectively. For each Nigel blue sky (Sun above the horizon) and twilight (Sun not more than $5^{\circ}$ below the horizon) observation, the equivalent widths (EWs) of both bands, $\mathrm{EW}_{\alpha}$ and $\mathrm{EW}_{\beta}$, were measured (Brault et al. 1975) by first approximating the continuum-essentially the blackbody

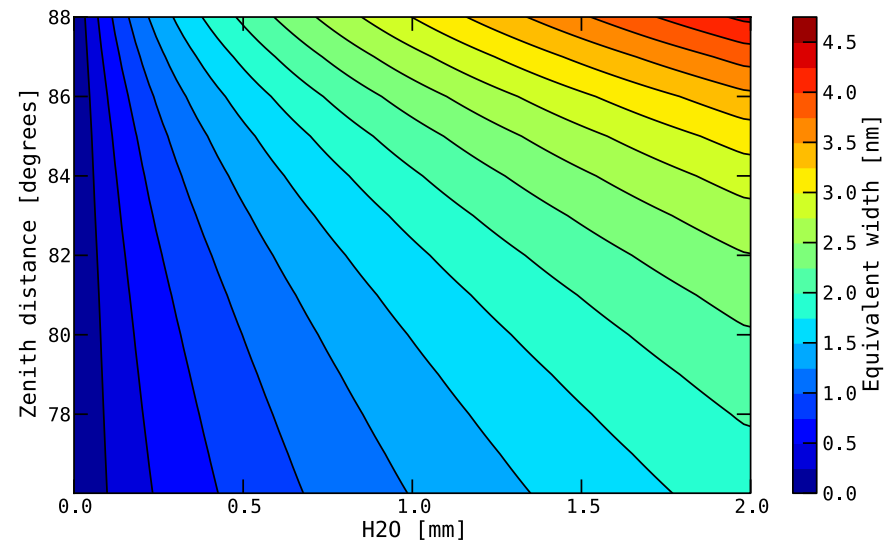

FIG. 4.-Relationship between solar zenith distance $\left(\zeta_{\odot}\right)$, equivalent width $\left(\mathrm{EW}_{\beta}\right)$, and PWV from the diffuse irradiance model. See the electronic edition of the PASP for a color version of this figure. 


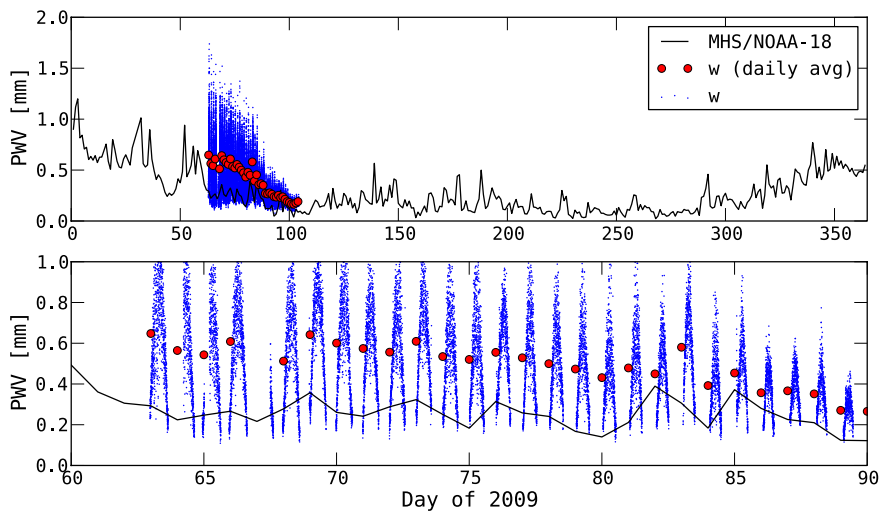

FIG. 5.-PWV estimates from Nigel using the Bird \& Riordan (1986) diffuse irradiance model. See the electronic edition of the PASP for a color version of this figure.

spectrum of the Sun - with a polynomial curve of order three. A transmissionlike spectrum was produced by dividing through by the continuum, from which the EW was then derived. A sample observation is presented in Figure 3. In a similar manner the equivalent widths of the Fraunhofer absorption bands of molecular oxygen, $\mathrm{EW}_{A}$ and $\mathrm{EW}_{B}$, were also extracted.

The analysis presented here was performed with data obtained in 2009 from Nigel's zenith field, with a focus on data from day numbers $\sim 60-120$. The day range was selected to begin with Nigel's first light around March and to end when it was no longer possible to obtain a bright twilight spectrum (i.e., the Sun was constantly $5^{\circ}$ or more below the horizon). Despite the frequency of Nigel observations, the results that follow are ultimately collapsed into daily averages in order to match the relative infrequent satellite passes, which provide the only benchmark for comparison.

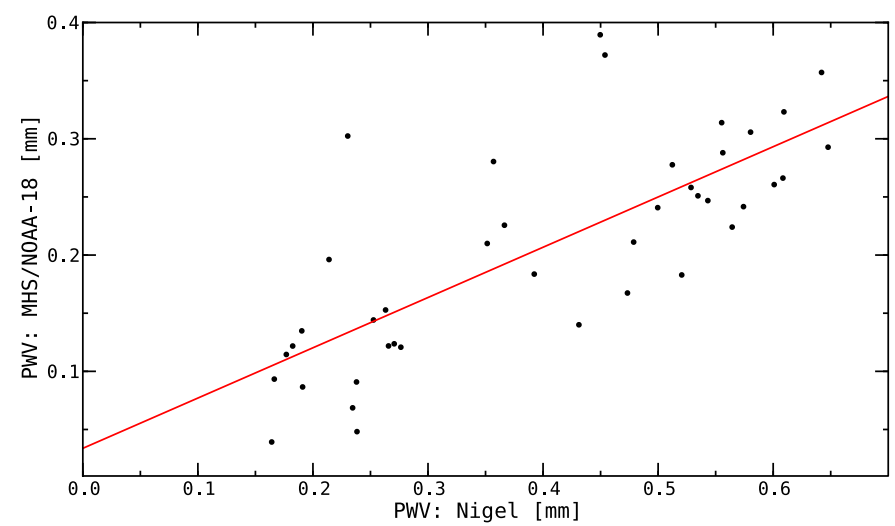

FIG. 6.-Correlation of daily average PWV as determined with Nigel using the diffuse irradiance model, with the MHS/NOAA-18 values. The equation of the line is $y=0.43 x+0.03$. See the electronic edition of the PASP for a color version of this figure.

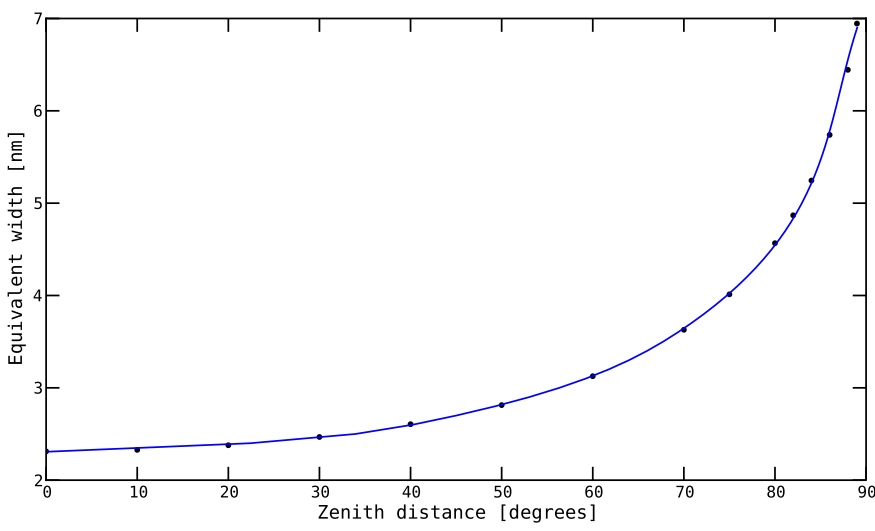

FIG. 7.-Oxygen A-band curve of growth. See the electronic edition of the $P A S P$ for a color version of this figure.

\section{PWV EXTRACTION USING NIGEL}

\subsection{Diffuse Irradiance Model}

One approach to obtain the PWV from diffuse sky spectra is to model the diffuse irradiance analytically. The most complete model available is that of Bird \& Riordan (1986), which uses algorithms to model the spectral irradiance at low $(10 \mathrm{~nm})$ resolution. The formulae have been rigorously tested and tweaked against full radiative transfer codes such as BRITE (Bird 1981), as well as field measurements, and they include the contributions due to both Rayleigh and Mie scattering. There are a number of input parameters for the model; Table 1 lists those parameters that are considered to be fixed (i.e., do not affect the water absorption bands within the scope of the model), with values chosen to be appropriate for the Antarctic plateau.

The other primary inputs are the tilt (chosen to be 0 for a horizontal surface; i.e., looking at the zenith), aspect (chosen arbitrarily to be north), solar zenith distance (via the date and time of the observation), and the PWV content. The model

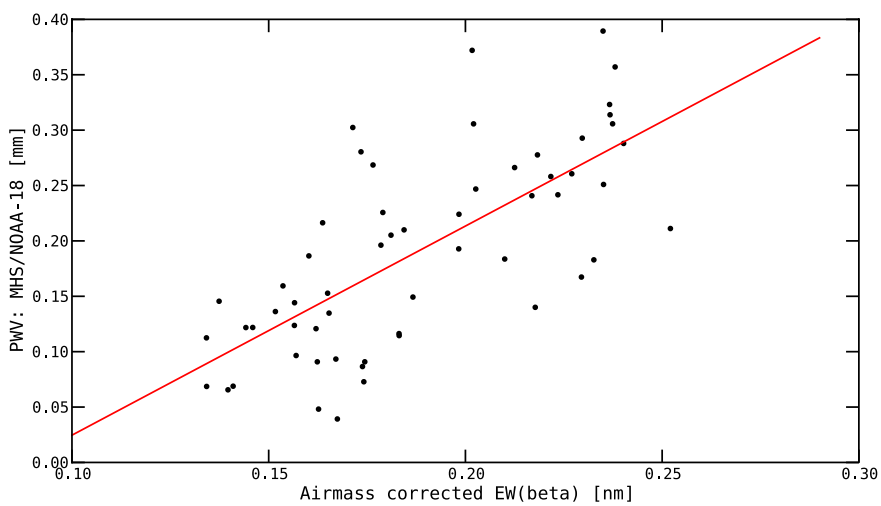

FIG. 8. Correlation of air-mass-corrected $\mathrm{EW}_{\beta}$ with MHS/NOAA-18 PWV values. The equation of the line is $y=1.89 x-0.16$. See the electronic edition of the PASP for a color version of this figure. 


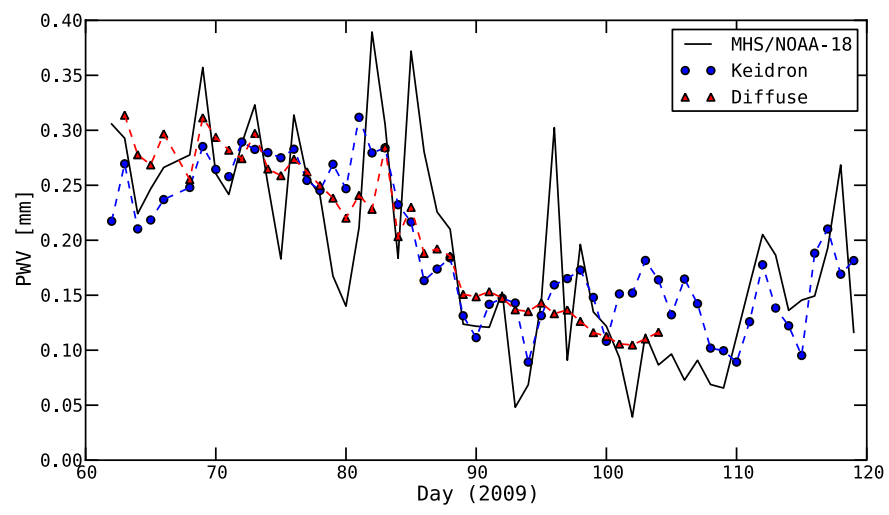

FIG. 9.-Comparison of satellite and Nigel PWV estimates from diffuse model and Keidron method. The Nigel values have been corrected with an offset and multiplicative factor. See the electronic edition of the $P A S P$ for a color version of this figure.

was run using a $\mathrm{C}$ implementation ${ }^{20}$ that was modified to allow batch processing of the input parameters.

A grid of models was produced for solar zenith distances $\left(\zeta_{\odot}\right.$ ) from $74-89^{\circ}$ (in $1^{\circ}$ increments) and with PWV values from 0-2 $\mathrm{mm}$ (in $0.1 \mathrm{~mm}$ increments). The continuum spectrum was estimated by setting the water content to zero, and the equivalent widths, $\mathrm{EW}_{\alpha}$ and $\mathrm{EW}_{\beta}$, were then calculated (computed at the same $\zeta_{\odot}$ ). The relationship is presented in Figure 4. One limitation of the model is that it is only valid for $\zeta_{\odot}<90^{\circ}$.

Using the gridded data points and 2D interpolation, the PWV content was calculated as a function of the measured equivalent widths and known solar zenith distance at the time of each observation, giving two estimates for the PWV $-w_{\alpha}$ and $w_{\beta}$. The final estimate, $w$, was taken to be the average of the two values. Figure 5 presents $w$ and the values as measured by MHS/ NOAA-18. The daily average values of $w$ are also shown.

The model predicts a physically unrealistic large diurnal effect, driven by the model's strong dependence of EW on solar zenith distance. It remains unclear as to the reason for such a large divergence, since only a small $(<5 \%)$ diurnal variation is expected (P. Ricaud 2011, private communication). Daily averages, however, while clearly overestimating the actual PWV, are of correct order of magnitude and appear to follow the same trends as the satellite measurements. The rms error between the raw daily averages and MHS values is $0.22 \mathrm{~mm}$. Figure 6 shows the actual correlations of $w$ with the values from MHS/NOAA-18. The equation of the line obtained using an unconstrained leastsquares fit is $y=0.43 x+0.03$, indicating that the diffuse model has a wet bias of $0.03 \mathrm{~mm}$, and it overestimates the PWV by almost a factor of 2 . The data show a clear correlation $\left(R^{2}=0.57\right)$ and a resultant rms error of $0.06 \mathrm{~mm}$ or $\sim 10-50 \%$ of typical values from the sample period.

\footnotetext{
${ }^{20}$ See http://rredc.nrel.gov/solar/models/spectral/spectrl2/.
}

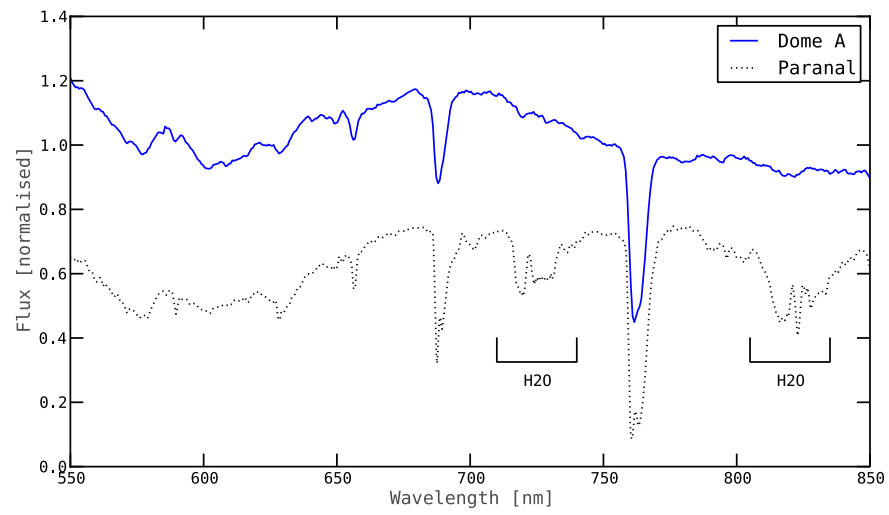

FIG. 10.-Comparison of Dome A and Paranal twilight sky spectra. The lack of $\mathrm{H}_{2} \mathrm{O}$ absorption in the Dome A spectrum is evident. Both spectra were obtained while the Sun was $\sim 1.4^{\circ}$ below the horizon. Dome A spectrum obtained with Nigel on 2009 April 4. Paranal spectrum were obtained with FORS1 on 2003 October 30 and adapted from Patat et al. (2006). The Dome A spectrum has been vertically offset by +0.3 for presentation. See the electronic edition of the $P A S P$ for a color version of this figure.

\subsection{Kiedron Method}

As a second approach to determining the PWV from Nigel spectra, we follow the method of Kiedron et al. (2003). The method builds on the premise that the EWs of water absorption features of a direct source are related to the column abundance of water vapor by a curve of growth. The additional and unknown amount of scattering present in diffuse irradiance is taken into account by introducing a new quantity called the "effective diffuse air mass." The effective diffuse air mass, based on the observed depth of the Fraunhofer oxygen A band at $759.4 \mathrm{~nm}$, represents the air mass at which you would expect the observation to have taken place in the absence of any scattering (i.e., observing direct irradiance). Using a precalculated curve of growth relating the EW of the oxygen A band to zenith distance for the

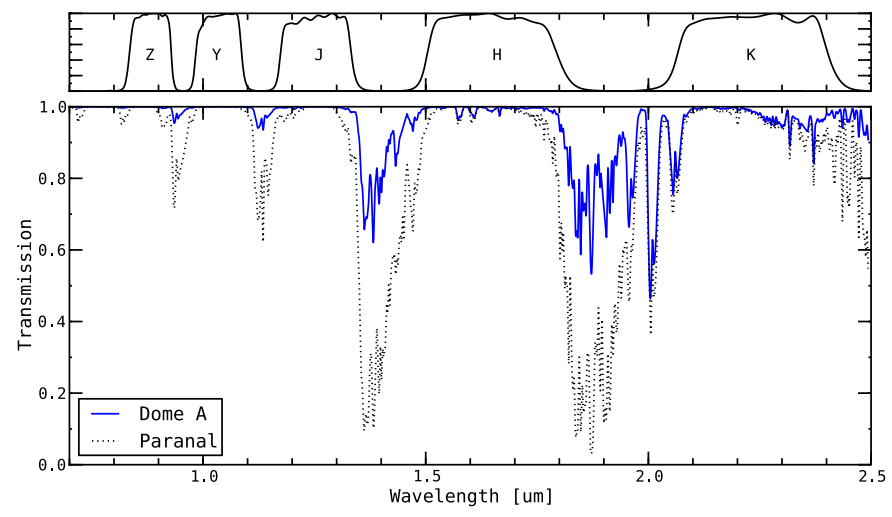

FIG. 11.-Atmospheric transmission of Dome A and Paranal using median PWV values. The spectrum is presented at low $(3 \mathrm{~nm})$ resolution for clarity. Curves on the upper plot trace the normalized $Z Y J H K$ photometric passbands (Hewett et al. 2006). See the electronic edition of the PASP for a color version of this figure. 

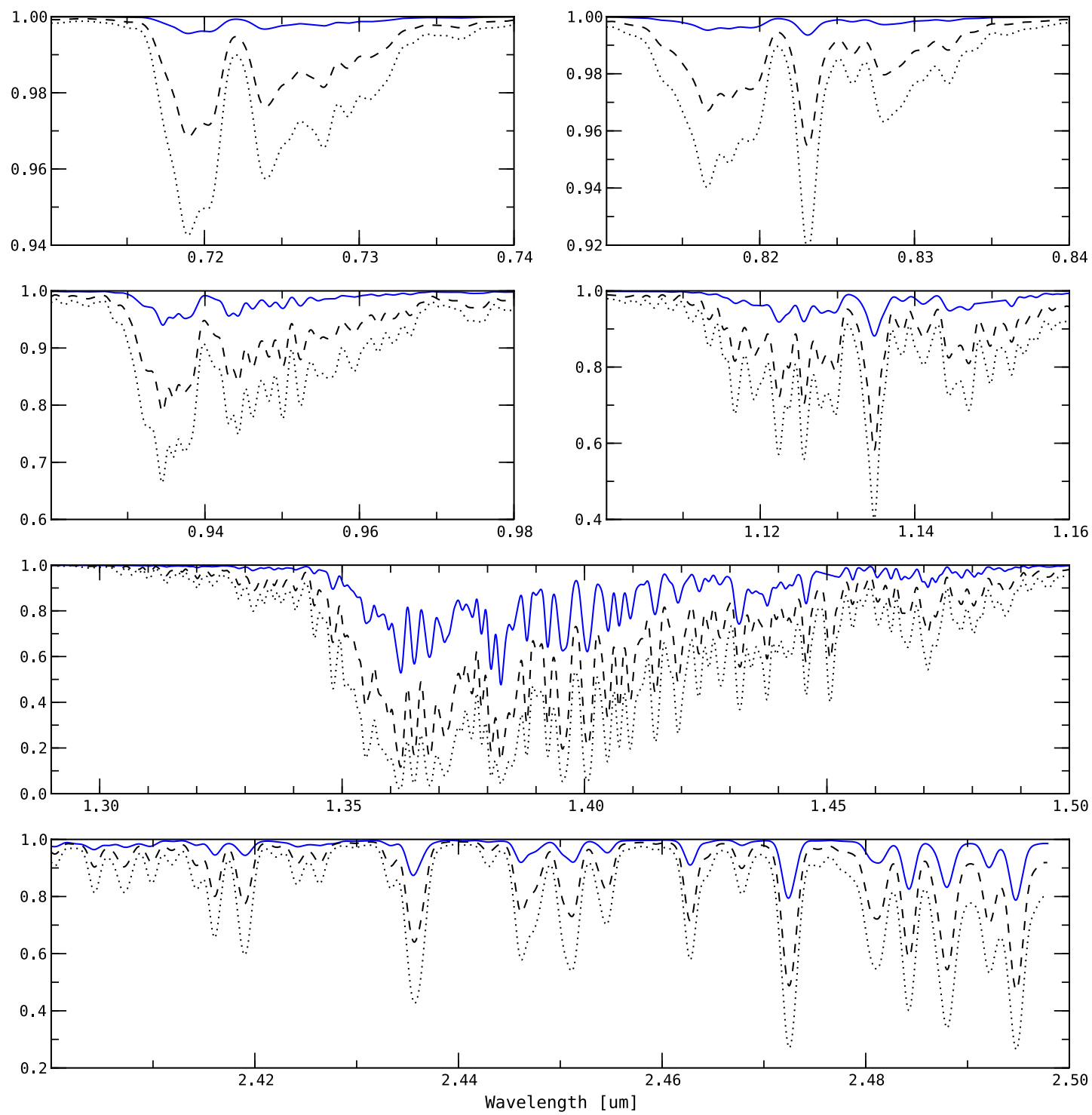

FIG. 12.-Atmospheric transmission of Dome A (solid line), Chajnantor (dashed line), and Paranal (dotted line) using median PWV values. The plots zoom in on the various atmospheric $\mathrm{H}_{2} \mathrm{O}$ bands at $1 \mathrm{~nm}$ resolution. Note the varying wavelength and transmission scales used. See the electronic edition of the PASP for a color version of this figure.

direct case, the measured EW from the diffuse spectrum is used as input to obtain the effective diffuse zenith distance, and hence effective diffuse air mass, of the observation.

The effective diffuse air mass is then used to scale the measured EW of the $\mathrm{H}_{2} \mathrm{O}$ bands in the diffuse spectra. Since the amount of oxygen is assumed to be constant, this effectively removes any dependency on the solar zenith distance or the amount of scattering that occurred. In theory, the scaled EW should then be directly proportional to the column-integrated water vapor.

One area in which our method differs from that of Kiedron et al. (2003) is that we use equivalent width as a measure of the depth of the absorption features, rather than the transmission at the center of the absorption feature. Second, Kiedron et al. use an empirically derived curve of growth for determining the effective diffuse air mass, utilizing side-by-side observations of the direct solar irradiance. These observations do not exist at our site, so instead a curve of growth was modeled using SpectralCalc. ${ }^{21}$ This curve of growth, shown in Figure 7, allows us to find the effective diffuse zenith distance (and hence air mass), given the measured $\mathrm{EW}$ of the oxygen $\mathrm{A}$ band, $\mathrm{EW}_{A}$.

The equivalent width measurements of interest, $\mathrm{EW}_{\beta}$ and $\mathrm{EW}_{A}$, were collapsed into daily averages. The effective diffuse

\footnotetext{
${ }^{21}$ See http://www.spectralcalc.com.
} 

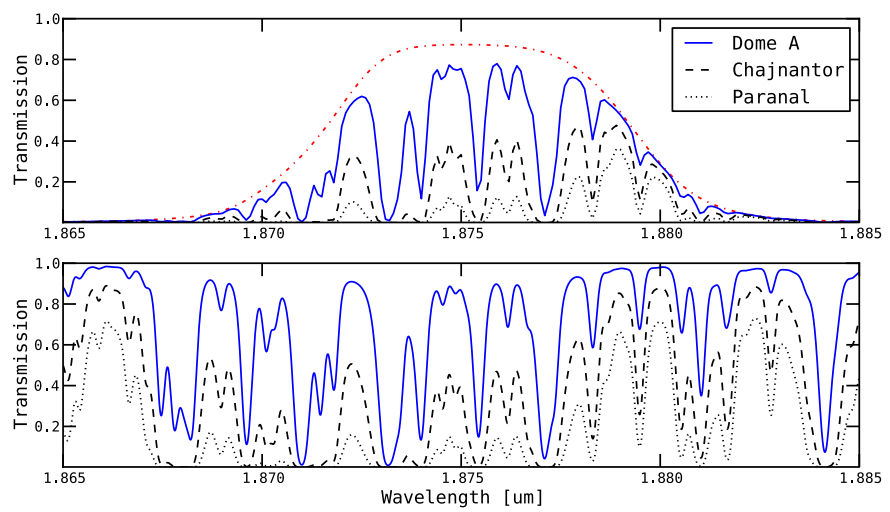

FIG. 13.-Bottom: Transmission of Dome A, Chajnantor, and Paranal around the Pa $\alpha$ emission line at $1.875 \mu \mathrm{m}$, using median PWV values. Top: N1875 filter profile (dash-dotted line) and the effective filter profiles at each site. The spectrum is presented at $1.5 \AA$ resolution to match similar simulations in Motohara et al. (2010). See the electronic edition of the PASP for a color version of this figure.

zenith distance was found using the curve of growth in Figure 7, which was then converted into effective diffuse air mass using the standard Kasten \& Young (1989) equation. The air-masscorrected equivalent width correlation with satellite PWV values is presented in Figure 8. The best-fit line has an equation $y=1.89 x-0.16$.

Once again a correlation is evident $\left(R^{2}=0.50\right)$, with a resultant rms error associated with the empirically determined relationship of $0.06 \mathrm{~mm}$ (10-50\% over the measured range), coincidently the same as that observed using the diffuse irradiance model. The correlation is not as strong as that observed by Kiedron et al. (2003), who reported $R^{2}=0.98$ and $\mathrm{rms}=0.23 \mathrm{~mm}$. While their rms error appears much higher, it is just $5-25 \%$ of their range of PWV values (1-5 mm).

A summary of the two methods is presented in Figure 9, where the PWV as derived from Nigel (and corrected with the MHS/NOAA-18 values) is plotted against the satellite values. The corrections involve an offset and multiplicative factor, as determined through the linear best-fit equations for each respective method.

TABLE 2

PWV Comparison at Dome A, Chajnantor, and Paranal

\begin{tabular}{ccccc}
\hline \hline \multicolumn{1}{c}{ Site } & $\begin{array}{c}\text { Altitude } \\
(\mathrm{m})\end{array}$ & $\begin{array}{c}\text { Median } \\
(\mathrm{mm})\end{array}$ & $\begin{array}{c}\text { Best 25\% } \\
(\mathrm{mm})\end{array}$ & $\begin{array}{c}\text { Best 10\% } \\
(\mathrm{mm})\end{array}$ \\
\hline Dome A, Antarctica $^{\mathrm{a}} \ldots \ldots$ & 4083 & 0.13 & 0.09 & 0.06 \\
Chajnantor, Chile $^{\mathrm{b}} \ldots \ldots \ldots$ & 5640 & 1.13 & 0.50 & 0.42 \\
Paranal, Chile $^{\mathrm{c}} \ldots \ldots \ldots \ldots$ & 2635 & 2.30 & 1.60 & 1.07 \\
\hline
\end{tabular}

${ }^{a}$ MHS/NOAA-18, during 2009 winter (days 120-300).

${ }^{\mathrm{b}}$ Erasmus \& van Staden (2001, unpublished) and Erasmus (2002; http:// www.eso.org/gen-fac/pubs/astclim/espas/radioseeing/pdf/ALMAFnlRep_ Erasmus_Dec2002.pdf), during 1993-1999.

${ }^{\mathrm{c}}$ Kerber et al. (2010), during 2001-2008.

\section{ATMOSPHERIC TRANSMISSION}

The extremely dry atmosphere above Dome A gives rise to an increased atmospheric transmission relative to temperate sites. While this has received extensive discussion as it relates to submillimeter and mid-infrared observations (e.g., Yang et al. 2010; Tremblin et al. 2011), its effect in the optical and near-IR spectral regions has not. The advantages conferred at these shorter wavelengths can be substantial. For example, the lack of absorption due to $\mathrm{H}_{2} \mathrm{O}$ can be clearly seen in a comparison of typical twilight sky spectra taken from Dome A and Paranal (a midlatitude observatory), shown in Figure 10. While previous authors have speculated on the potential gains in the optical/ near-IR (e.g., Lawrence et al. 2009b), we are now in a position to quantify these advantages.

To examine the benefits at these wavelengths, the atmospheric transmission in the zenith direction was modeled from $700 \mathrm{~nm}$ to $2.5 \mu \mathrm{m}$. The transmission spectrum for Dome A was compared with that of the Paranal and Chajnantor observatories in the Atacama desert, Chile, using the median ${ }^{22} \mathrm{PWV}$ value for each site. Spectra were modeled using SpectralCalc, which uses the HITRAN 2008 database (Rothman et al. 2009) and an online interface of the LINEPAK algorithm (Gordley et al. 1994) for modeling spectral transmittance and radiance. PWV amounts were specified by varying the water content of standard atmospheres (polar winter for Dome A; midlatitude winter for Chajnantor and Paranal), for which the PWV at specific altitudes had been precalculated. Figures 11, 12, and 13 show the modeled transmission. The actual median PWV values, along with the best 25 percentile and 10 percentile amounts for each site, are compared in Table 2.

The atmospheric models demonstrate that the transparency in the optical and near-IR regions at Dome A is far superior to that of even the highest and driest midlatitude observatories. With such improved transmission, observations are no longer limited to the standard photometric bands (which are defined largely to avoid water vapor absorption).

Cool stars and planets, for example, contain a host of metallic and molecular lines that appear in the near-IR spectrum (e.g., Kleinmann \& Hall 1986; Hinkle et al. 1989; Wallace \& Hinkle 1997; Joyce et al. 1998; Lançon \& Wood 2000; Lyubchik et al. 2004), some of which inevitably appear within the atmospheric $\mathrm{H}_{2} \mathrm{O}$ bands. One example is the $\mathrm{CH}_{4}$ icosad region (1.30$1.49 \mu \mathrm{m})$, which is of particular importance to the study of Saturn's moon Titan (Boudon et al. 2009; Campargue et al. 2010). In general, a dry atmosphere such as Dome A would allow a more complete interpretation of stellar spectra without such a strong dependence on the removal of telluric $\mathrm{H}_{2} \mathrm{O}$ absorption (Lançon et al. 2007), ultimately leading to a better

\footnotetext{
${ }^{22}$ In the optical/near-IR, only nighttime observations are usable, so the median is calculated on a year-round basis at Paranal and Chajnantor, but for only six months during winter (days 120-300) at Dome A.
} 
understanding of stellar evolution and improved stellar models (Lançon et al. 2009).

The hydrogen Paschen alpha $(\mathrm{Pa} \alpha)$ emission line (at $1.875 \mu \mathrm{m})$, is another spectral feature that could be exploited at Dome $\mathrm{A}$. $\mathrm{Pa} \alpha$ emission is a reliable tracer of star formation rates in regions that are otherwise obscured by dust clouds, where traditional indicators (e.g., $\mathrm{H} \alpha$ ) are unsuitable due to the added extinction (Díaz-Santos et al. 2008). Until recently, $\operatorname{Pa} \alpha$ emissions had only been observed from space-based surveys (e.g., Wang et al. 2010, using the Near-Infrared Camera and Multi-Object Spectrometer on Hubble Space Telescope). The first ground-based detections were reported by Motohara et al. (2010) from Chajnantor mountain, using the miniTAO $1 \mathrm{~m}$ telescope and a narrowband N1875 filter. The initial success has prompted plans for a larger $(6.5 \mathrm{~m})$ telescope to be developed (Yoshii et al. 2010). Figure 13 shows the atmospheric transmission around the $\mathrm{Pa} \alpha$ line, once again emphasizing the advantages of Dome A compared with Chajnantor, the site of the first ground-based $\mathrm{Pa} \alpha$ observation.

The three Antarctic Schmidt Telescopes (AST3) are the first planned instruments to utilize the unique near-IR conditions found at Dome A. Functioning as an array, the three $50 \mathrm{~cm}$ telescopes are intended to operate in the 400-900 nm range $(g, r$, and $i$ filters), each with one unique filter to enable simultaneous multicolor observations (Cui et al. 2008; Yuan et al. 2010). Science goals of AST3 include studying Type Ia supernovae and dark energy, microlensing and searching for extrasolar planets, searching for variable stars, asteroseismology, and general site testing. Deployment for AST3 is scheduled to begin in the 2011-2012 summer (Wang et al. 2012, in preparation).

It is worth noting that although Dome A is the driest established site on the Antarctic plateau, other stations such as Dome C and Dome F still boast lower PWV values (and hence increased transmission) than any midlatitude site (Saunders et al. 2009). As previously noted, the benefits of such conditions extend into the submillimeter (or terahertz) regime, but are beyond the scope of this article.

\section{CONCLUSION}

Diffuse optical sky spectra from Nigel have provided another technique to ground-truth satellite observations and yet again verify the extremely low atmospheric water vapor content at Dome A. The corrected daily averages as derived from Nigel using the diffuse irradiance model agree with the MHS/ NOAA-18 satellite values to within $\pm 50 \%$. The Keidron analysis produced an empirical relationship that allows PWV determinations from Nigel with similar accuracy. Relative infrequent satellite passes, compared with the Nigel observation frequency, prohibit a more detailed analysis (higher temporal resolution) from being undertaken. Additionally, both methods are limited to times when the Sun is above (diffuse model) or no more than $5^{\circ}$ below (Keidron method) the horizon.

The increased transmission at Dome A on the Antarctic plateau, resulting from extremely dry atmospheric conditions, creates a near-continuous atmospheric window in the optical to near-IR spectral regime, unrivaled anywhere else on Earth.

This research is supported by the Chinese PANDA International Polar Year project and the Polar Research Institute of China. The authors wish to thank all the members of the 2008/2009/2010 Polar Research Institute of China Dome A expeditions for their heroic efforts in reaching the site and for providing invaluable assistance to the expedition astronomers in setting up PLATO and its associated instrument suite. This research is financially supported by the Australian Research Council, the Australian Antarctic Division, the Chinese Academy of Sciences, the National Natural Science Foundation of China, the US National Science Foundation, and the United States Antarctic Program. Additional financial contributions have been made by the institutions involved in this collaboration. We are grateful to Ferdinando Patat, Florian Kerber, and Kentaro Motohara for providing data files from their respective publications, which were used for a number of plots and tables in this article. We also thank Jeremy Bailey for helpful discussions.

\section{REFERENCES}

Aumann, H. H., et al. 2003, IEEE Trans. Geosci. Rem. Sens., 41, 253

$\rightarrow$ Bevis, M., Businger, S., Chiswell, S., Herring, T. A., Anthes, R. A., Rocken, C., \& Ware, R. H. 1994, J. Appl. Meteorol., 33, 379

Bevis, M., Businger, S., Herring, T. A., Rocken, C., Anthes, R. A., \& Ware, R. H. 1992, J. Geophys. Res., 971, 15787

Bird, R. E. 1981, NASA STI/Recon Tech. Rep. N (Washington: NASA), 83, 25104

$\rightarrow$ Bird, R. E., \& Riordan, C. 1986, J. Appl. Meteorol., 25, 87

$\rightarrow$ Bonsignori, R. 2007, Proc. SPIE, 6744, 67440A

$\rightarrow$ Boudon, V., et al. 2009, Europhys. News, 40, 17

$\rightarrow$ Brault, J. W., Fender, J. S., \& Hall, D. N. B. 1975, J. Quant. Spec. Radiat. Transf., 15, 549

$\rightarrow$ Campargue, A., Wang, L., Kassi, S., Mašát, M., \& Votava, O. 2010, J. Quant. Spec. Radiat. Transf., 111, 1141

$\rightarrow$ Cui, X., Yuan, X., \& Gong, X. 2008, Proc. SPIE, 7012, 70122D $\rightarrow$ Dempsey, J. T., Storey, J. W. V., Ashley, M. C. B., Burton, M. G., Calisse, P. G., \& Jarnyk, M. A. 2004, Proc. SPIE, 5492, 811

$\rightarrow$ Díaz-Santos, T., Alonso-Herrero, A., Colina, L., Packham, C., Radomski, J. T., \& Telesco, C. M. 2008, ApJ, 685, 211

Emrich, A., et al. 2009, in Twentieth Int. Symp. Space Terahertz Tech., ed. E. Bryerton, A. Kerr, \& A. Lichtenberger (Charlottesville: NRAO), 174

$\rightarrow$ Gordley, L. L., Marshall, B. T., \& Chu, D. A. 1994, J. Quant. Spec. Radiat. Transf., 52, 563

$\rightarrow$ Halthore, R. N., Eck, T. F., Holben, B. N., \& Markham, B. L. 1997, J. Geophys. Res., 102, 4343

Hébert, P., et al. 2004, in 5th Int. Conf. Space Optics, ed. B. Warmbein (ESA SP-554, Noordwijk: ESA), 49

$\rightarrow$ Hewett, P. C., Warren, S. J., Leggett, S. K., \& Hodgkin, S. T. 2006, MNRAS, 367, 454 
Hinkle, K. H., Lambert, D. L., \& Wing, R. F. 1989, MNRAS, 238,1365

$\rightarrow$ Joyce, R. R., Hinkle, K. H., Wallace, L., Dulick, M., \& Lambert, D. L. 1998, AJ, 116, 2520

$\rightarrow$ Kasten, F., \& Young, A. T. 1989, Appl. Opt., 28, 4735

$\rightarrow$ Kenyon, S. L., Ashley, M. C. B., Everett, J., Lawrence, J. S., \& Storey, J. W. V. 2006, Proc. SPIE, 6267, 62671M

$\rightarrow$ Kerber, F., et al. 2010, Proc. SPIE, 7733, 77331M

$\rightarrow$ Kiedron, P., Berndt, J., Michalsky, J., \& Harrison, L. 2003, Geophys. Res. Lett., 30, 1565

$\rightarrow$ Kleinmann, S. G., \& Hall, D. N. B. 1986, ApJS, 62, 501

Kulesa, C. A., et al. 2008a, in Ninteenth Int. Symp. Space Terahertz Tech., ed. W. Wild (Charlottesville: NRAO), 390

$\rightarrow$ Kulesa, C. A., et al. 2008b, Proc. SPIE, 7012, 701249

$\rightarrow$ Laine, V. 2008, Rem. Sens. Env., 112, 646

$\rightarrow$ Lançon, A., Gallagher, J. S., Mouhcine, M., Smith, L. J., Ladjal, D., \& de Grijs, R. 2009, Ap\&SS, 324, 241

$\rightarrow$ Lançon, A., Hauschildt, P. H., Ladjal, D., \& Mouhcine, M. 2007, A\&A, 468, 205

$\rightarrow$ Lançon, A., \& Wood, P. R. 2000, A\&AS, 146, 217

$\rightarrow$ Lawrence, J. S. 2004, PASP, 116, 482

$\rightarrow$ Lawrence, J. S., Ashley, M. C. B., Hengst, S., Luong-van, D. M., Storey, J. W. V., Yang, H., Zhou, X., \& Zhu, Z. 2009a, Rev. Sci. Instrum., 80, 064501

$\rightarrow$ Lawrence, J. S., et al. 2009b, PASA, 26, 379

$\rightarrow$ Lyubchik, Y., Jones, H. R. A., Pavlenko, Y. V., Viti, S., Pickering, J. C., \& Blackwell-Whitehead, R. 2004, A\&A, 416, 655

$\rightarrow$ Miao, J., Kunzi, K., Heygster, G., Lachlan-Cope, T. A., \& Turner, J. 2001, J. Geophys. Res., 106, 10187

$\rightarrow$ Motohara, K., et al. 2010, Proc. SPIE, 7735, 77353K

$\rightarrow$ Patat, F., Ugolnikov, O. S., \& Postylyakov, O. V. 2006, A\&A, 455, 385

$\rightarrow$ Patat, F., et al. 2011, A\&A, 527, A91

$\rightarrow$ Querel, R. R., Naylor, D. A., \& Kerber, F. 2011, PASP, 123, 222 $\rightarrow$ Querel, R. R., Naylor, D. A., Thomas-Osip, J., Prieto, G., \& McWilliam, A. 2008, Proc. SPIE, 7014, 701457

$\rightarrow$ Raj, P. E., Devara, P. C. S., Maheskumar, R. S., Pandithurai, G., Dani, K. K., Saha, S. K., Sonbawne, S. M., \& Tiwari, Y. K. 2004, J. Appl. Meteorol., 43, 1452

$\rightarrow$ Ricaud, P., Gabard, B., Derrien, S., Chaboureau, J.-P., Rose, T., Mombauer, A., \& Czekala, H. 2010, IEEE Trans. Geosci. Rem. Sens., 48, 1365

Ricaud, P., et al. 2011, IEEE Trans. Geosci. Rem. Sens., submitted

$\rightarrow$ Rothman, L. S., et al. 2009, J. Quant. Spec. Radiat. Transf., 110, 533

Rozenberg, G. V. 1966, Twilight-A Study in Atmospheric Optics (New York: Plenum)

$\rightarrow$ Saunders, W., et al. 2009, PASP, 121, 976

$\rightarrow$ Schlüssel, P., Hultberg, T. H., Phillips, P. L., August, T., \& Calbet, X. 2005, Adv. Space Res., 36, 982

$\rightarrow$ Sims, G., et al. 2010, Proc. SPIE, 7733, 77334M

$\rightarrow$ Six, D., Fily, M., Blarel, L., \& Goloub, P. 2005, Atmos. Env., 39, 5041 Stone, R. S. 2002, in 9th Workshop Italian Research on Antarctic Atmosphere (Bologna: Italian Phys. Soc.), 80, 123

Storey, J. W. V., et al. 2011, Bull. Astron. Soc. India, submitted

$\rightarrow$ Suparta, W., Abdul Rashid, Z. A., Mohd. Ali, M. A., Yatim, B., \& Fraser, G. J. 2008, J. Atmos. Sol.-Terr. Phys., 70, 1419

$\rightarrow$ Susskind, J., Blaisdell, J., \& Iredell, L. 2009, Proc. SPIE, 7453, 74530F

$\rightarrow$ Thomas, I. D., King, M. A., Clarke, P. J., \& Penna, N. T. 2011, J. Geophys. Res. Atmos., 116, D04107

$\rightarrow$ Tremblin, P., et al. 2011, A\&A, 535, A112

$\rightarrow$ Wallace, L., \& Hinkle, K. 1997, ApJS, 111, 445

$\rightarrow$ Wang, Q. D., et al. 2010, MNRAS, 402, 895

Wiedner, M., Hills, R., \& Pardo-Carrión, J. 2002, in ASP Conf. Ser. 266, Astronomical Site Evaluation in the Visible and Radio Range (San Francisco: ASP), 278

$\rightarrow$ Yang, H., et al. 2010, PASP, 122, 490

$\rightarrow$ Yoshii, Y., et al. 2010, Proc. SPIE, 7733, 773308

$\rightarrow$ Yuan, X., et al. 2010, Proc. SPIE, 7733, 77331V 